\title{
उMR
}

\section{Upregulation of fibroblast growth factor 1 in the synovial membranes of patients with late stage osteoarthritis}

\author{
R. Li ${ }^{1,2 *}$, B. Wang ${ }^{3,4 *}$, C.Q. He ${ }^{1}$, Y.Q. Yang ${ }^{5}$ H. Guo ${ }^{1}$, Y. Chen ${ }^{1}$ and T.H. Du ${ }^{1}$ \\ ${ }^{1}$ Department of Rehabilitation, West China Hospital, Sichuan University, \\ Chengdu, Sichuan, China \\ ${ }^{2}$ Department of Rehabilitation, Guizhou Provincial People's Hospital, \\ Guiyang, Guizhou, China \\ ${ }^{3}$ Department of Eugenics and Genetics, \\ Guiyang Maternal and Child Health-Care Hospital, Guiyang, Guizhou, China \\ ${ }^{4}$ College of Medical Examination, Guiyang Medical University, Guiyang, China \\ ${ }^{5}$ Department of Orthopaedic Surgery, Affiliated Hospital of Guiyang Medical \\ College, Guiyang Medical University, Guiyang, China \\ *These authors contributed equally to this study \\ Corresponding author: C.Q. He \\ E-mail: chengqi_he@yeah.net \\ Genet. Mol. Res. 14 (3): 11191-11199 (2015) \\ Received January 14, 2015 \\ Accepted May 14, 2015 \\ Published September 22, 2015 \\ DOI http://dx.doi.org/10.4238/2015.September.22.13
}

\begin{abstract}
Osteoarthritis (OA) is a degenerative disease of the systemic joint that involves multiple cytokines and growth factors. Fibroblast growth factor 1 (FGF-1) is increased in patients with rheumatic arthritis. The aim of this study was to determine whether the expression and secretion of FGF-1 differed in synovial tissue from patients with late stage OA from that in normal tissues. We selected eight patients with late stage OA and eight healthy donors for this study. An enzyme-linked immunosorbent assay was used to determine the amount of FGF-1 in the synovial fluid and in the culture medium of synovial fibroblasts. Real time quantitative polymerase chain reaction
\end{abstract}


(qPCR) analysis was performed to examine the expression levels of $F G F-1$ and FGF receptor 2 (FGFR2) in synovial and cartilage tissues. We detected FGF-1 in the synovial fluid from all eight donors, as well as in the culture medium of synovial fibroblasts. Synovial fluid from patients with OA and culture medium of OA synovial fibroblasts contained significantly more FGF-1 than those from controls. FGF-1 expression was also lower in the synovial membranes of normal donors than in those of OA patients. FGFR2 expression was also higher in OA cartilage than in normal cartilage. Overall, these results demonstrated that FGF-1 synthesis and secretion by synovial fibroblasts were significantly increased in OA. FGFR2 expression was also shown to be upregulated in patients with $\mathrm{OA}$. These findings suggest that increased FGF-1 signaling correlates with an OA pathological condition.

Key words: Fibroblast growth factor 1; Osteoarthritis; Cartilage; Chondrocytes

\section{INTRODUCTION}

Osteoarthritis (OA) is recognized as a degenerative disease of the systemic joint that often results from cartilage damage. OA progression normally causes functional impairment and eventually leads to joint disability (Zhen and Cao, 2014). Cartilage degradation during OA involves a group of catabolic factors such as interleukin (IL)-1 and matrix metalloproteinases (MMPs) (Daheshia and Yao, 2008; Berenbaum, 2013). Blockage of these factors shows some therapeutic benefit by attenuating the degeneration of cartilage. However, inhibiting one or two factors is not effective in general, since a large number of catabolic factors are involved in the degenerative process of cartilage tissue. Manipulation of the up-stream cytokines may, however, be reasonably considered as a more effective way to inhibit the catabolic factors in OA progression. Fibroblast growth factor 1 (FGF-1) is often considered to be a potent angiogenic and neurotrophic factor. It belongs to the FGF super-family, which contains 22 members in humans and in mice (Itoh and Ornitz, 2004). FGF-1 is generally involved in the development of vertebrates at the embryonic and fetal stages (Böttcher and Nierhs, 2005). It is also believed to play important roles in normal physiological processes as well as in pathological conditions (Chen and Forough, 2006). FGF-1 activates a group of FGF receptors (FGFRs), all of which are tyrosine kinase cell-surface receptors. It is usually expressed and stored in cells under normal physiological conditions; however, when cells are under stress, FGF-1 can be secreted into the extracellular environment (Jackson et al., 1992). It has been reported that FGF-1 is synthesized by osteoblasts and chondrocytes throughout the healing process and it is believed to stimulate the proliferation, maturation, and matrix deposition of immature chondrocytes (Bolander 1992). In a more recent study, FGF-1 has been shown to be secreted by mesenchymal stem cells to accelerate the proliferation and matrix production of osteoarthritic chondrocytes in a co-culture model (Wu et al., 2013). To understand how the expression and regulation of FGF-1 is influenced in OA pathological conditions, we determined the concentrations of FGF-1 in synovial fluid from normal individuals and from patients with OA. The expression of $F G F-1$ and FGF receptors at the mRNA level was also examined in synovial and cartilage tissue from these two groups. Our study provides evidence for understanding the role of FGF-1 signaling in the pathology of OA. 


\section{MATERIAL AND METHODS}

\section{Cell culture and expansion}

Normal synovial membranes, synovial fluid, and cartilage of eight healthy human donors were obtained from the West China Hospital within $24 \mathrm{~h}$ post-mortem by dissecting knee joints. OA synovial membranes, synovial fluid, and cartilage were obtained from patients with end stage OA (eight donors) undergoing total knee replacement. Basic information for all donors is listed in Table 1. Both normal and OA synovial membranes were digested for 20-22 $\mathrm{h}$ in collagenase type I $(1 \mathrm{mg} / \mathrm{mL}$, Worthington, NJ, USA) dissolved in proliferation medium [alpha-MEM supplemented with 10\% fetal bovine serum (FBS), $0.2 \mathrm{mM}$ ascorbic acid 2-phosphate, $100 \mathrm{U} / \mathrm{mL}$ penicillin, and $100 \mu \mathrm{g} / \mathrm{mL}$ streptomycin]. Synovial fibroblasts were cultured in proliferation medium.

The use of human materials was approved by the local Medical Ethical Committee of Sichuan University. All patients participated in this study signed the informed consents.

\begin{tabular}{|c|c|c|c|c|}
\hline Donor & Age (years) & Gender & Joint & OA grade \\
\hline \multicolumn{5}{|l|}{ Normal } \\
\hline 1 & 78 & Female & Hip & 0 \\
\hline 2 & 69 & Female & Knee & 0 \\
\hline 3 & 45 & Male & Hip & 0 \\
\hline 4 & 72 & Male & Knee & 0 \\
\hline 5 & 80 & Female & Knee & 0 \\
\hline 6 & 81 & Female & Knee & 0 \\
\hline 7 & 75 & Female & Knee & 0 \\
\hline 8 & 68 & Male & Knee & 0 \\
\hline \multicolumn{5}{|l|}{$\mathrm{OA}$} \\
\hline 1 & 57 & Female & Knee & III \\
\hline 2 & 74 & Male & Knee & III \\
\hline 3 & 62 & Female & Knee & III \\
\hline 4 & 68 & Male & Knee & III \\
\hline 5 & 80 & Male & Hip & III \\
\hline 6 & 82 & Female & Hip & III \\
\hline 7 & 76 & Female & Hip & III \\
\hline 8 & 68 & Male & Knee & III \\
\hline
\end{tabular}

$\mathrm{OA}=$ osteoarthritis.

\section{Enzyme-linked immunosorbent assay (ELISA) assay}

A human FGF-1 ELISA kit (R\&D Systems, Minneapolis, MN, USA) was used to determine the concentration of active human FGF-1 in synovial fluids and in the culture medium of synovial fibroblasts according to the manufacturer instructions. Absorbance was measured on an ELISA plate reader (Infinite ${ }^{\circledR} 200$ PRO, Tecan, Switzerland) at $450 \mathrm{~nm}$. The absorbance value at $550 \mathrm{~nm}$ was also measured for subtraction from the absorbance value at $450 \mathrm{~nm}$ for correction of the optical imperfections in the microplates.

\section{Collection of synovial fibroblast culture medium}

Synovial fibroblasts were sub-cultured to passage 2 with $90 \%$ confluence. The culture 
medium of synovial fibroblasts was obtained by incubating synovial fibroblasts with serumfree alpha-MEM for $48 \mathrm{~h}$. Medium was then passed through a $0.22 \mathrm{~mm}$ filter, and stored at $-20^{\circ} \mathrm{C}$ until assayed.

\section{Immunofluorescence staining and image acquisition}

Synovial fibroblasts at passage 2 were plated on glass coverslips on six-well plates 24 $\mathrm{h}$ before staining. Cells were washed with phosphate buffered saline (PBS) and fixed in 4\% paraformaldehyde for $30 \mathrm{~min}$ at room temperature. Permeabilization was accomplished with $0.5 \%$ Triton-X 100. Bovine serum albumin (BSA) $(0.5 \%)$ was used for blocking. Coverslips were subsequently incubated at $37^{\circ} \mathrm{C}$ with rabbit polyclonal antibodies against FGF-1 (AbCam, Cambridge, MA, USA) for $2 \mathrm{~h}$. Then, coverslips were incubated with secondary antibodies conjugated to Alexa 488 (Invitrogen, Carlsbad, CA, USA). Nuclei were visualized with 4,6-diamidino-2-phenylindole (DAPI) (Molecular Probes, Eugene, OR, USA). The synovial membranes of patients with OA and normal donors were harvested for immunofluorescence staining. Tissue was freshly frozen before section. Cryo-sections of tissue were stained with the same primary and secondary antibodies as described above. Images were obtained using a DMi $6000 \mathrm{~B}$ fluorescent microscope (Leica, Bensheim, Germany) with the software accompanying the microscope.

\section{RNA isolation and quantitative polymerase chain reaction (qPCR)}

RNA from synovial membranes and cartilage tissue was isolated using an RNA purification kit (Tiangen, Beijing, China). Briefly, tissues were snap-frozen with liquid nitrogen and crushed by mortar and pestle. Then the homogenized tissue was lysed with a lysis buffer. Total RNA was isolated according to the manufacturer protocol. For each sample, $1 \mathrm{mg}$ total RNA was reverse-transcribed into cDNA using a cDNA Synthesis kit (TaKaRa, Dalian, China). qPCR was performed on cDNA samples by using the SYBR ${ }^{\circledR}$ Premix Ex Taq Real-Time PCR Kit (TaKaRa). PCRs were carried out on a MyiQ2 Two-Color Real-Time PCR Detection System (Bio-Rad, Hercules, CA, USA) under the following conditions: cDNA was denatured for $5 \mathrm{~min}$ at $95^{\circ} \mathrm{C}$, followed by 45 cycles of $15 \mathrm{~s}$ at $95^{\circ} \mathrm{C}, 15 \mathrm{~s}$ at $60^{\circ} \mathrm{C}$, and $30 \mathrm{~s}$ at $72^{\circ} \mathrm{C}$. For each reaction, a melting curve was generated to test for primer dimer formation and non-specific priming. The sequences of primers used for qPCR are listed in Table 2. Relative expression was calculated using the double delta $\mathrm{Ct}$ method (Livak and Schmittgen, 2001). Both GAPDH and $\beta$-actin primers were used for normalization. Following qPCR, all products were resolved on a $2 \%$ agarose gel and visualized with a fluorescent dye nucleic acid stain (SBS Genetic Technology, Shanghai, China).

\section{Statistical analysis}

Differences between the results from culturing normal and OA samples were examined for statistical significance with the Student $t$ - test. $\mathrm{P}$ values of $<0.05$ were considered as significant.

\section{RESULTS}

To understand how an OA pathological condition influences FGF-1 secretion, an ELISA was performed to determine the concentrations of FGF-1 in the synovial fluids of normal 
individuals and patients with OA. As shown in Figure 1A, synovial fluid from normal donors contained approximately $35 \mathrm{pg} / \mathrm{mL}$ FGF-1 with a range of concentrations from 3 to $60 \mathrm{pg} / \mathrm{mL}$ in the 8 donors tested. FGF-1 concentrations significantly increased to approximately $110 \mathrm{pg} /$ $\mathrm{mL}$ in the synovial fluid of patients with OA, varying from 80 to $190 \mathrm{pg} / \mathrm{mL}$. To confirm that the synovial tissue was the main source of FGF-1 in the synovial fluid, synovial fibroblasts were isolated and cultured in vitro, and the concentrations of FGF-1 in the culture medium of synovial fibroblasts from normal and OA tissues were measured (Figure 1B). The culture medium of OA synovial fibroblasts contained approximately $70 \%$ of the FGF-1 as did the OA synovial fluid, whereas the culture medium of normal synovial fibroblasts had similar levels of FGF-1 as did the normal synovial fluid, with an average concentration of approximately $40 \mathrm{ng} / \mathrm{mL}$. The difference between the normal and OA cultured medium was statistically significant $(\mathrm{P}=0.0015)$.

\begin{tabular}{|c|c|c|c|}
\hline Gene & Sequence (5' - 3') & Amplicon size & PrimerBank ID \\
\hline Fibroblast growth factor $1(F G F 1)$ & $\begin{array}{l}\text { Forward: } \\
\text { TTCACAGCCCTGACCGAGAA } \\
\text { Reverse: } \\
\text { CGTTGCTACAGTAGAGGAGTTTG }\end{array}$ & 76 & 15055541a1 \\
\hline Fibroblast growth factor receptor 2 (FGFR2) & $\begin{array}{l}\text { Forward: } \\
\text { AGCACCATACTGGACCAACAC } \\
\text { Reverse: } \\
\text { GGCAGCGAAACTTGACAGTG }\end{array}$ & 83 & $222144234 \mathrm{c} 1$ \\
\hline Beta actin & $\begin{array}{l}\text { Forward: } \\
\text { CATGTACGTTGCTATCCAGGC } \\
\text { Reverse: } \\
\text { CTCCTTAATGTCACGCACGAT }\end{array}$ & 250 & 4501885a1 \\
\hline Glyceraldehyde-3-phosphate dehydrogenase (GAPDH) & $\begin{array}{l}\text { Forward: } \\
\text { TGTGGGCATCAATGGATTTGG } \\
\text { Reverse: } \\
\text { ACACCATGTATTCCGGGTCAAT }\end{array}$ & 116 & $126273608 \mathrm{c} 1$ \\
\hline
\end{tabular}

$\mathrm{qPCR}=$ real-time quantitative polymerase chain reaction .
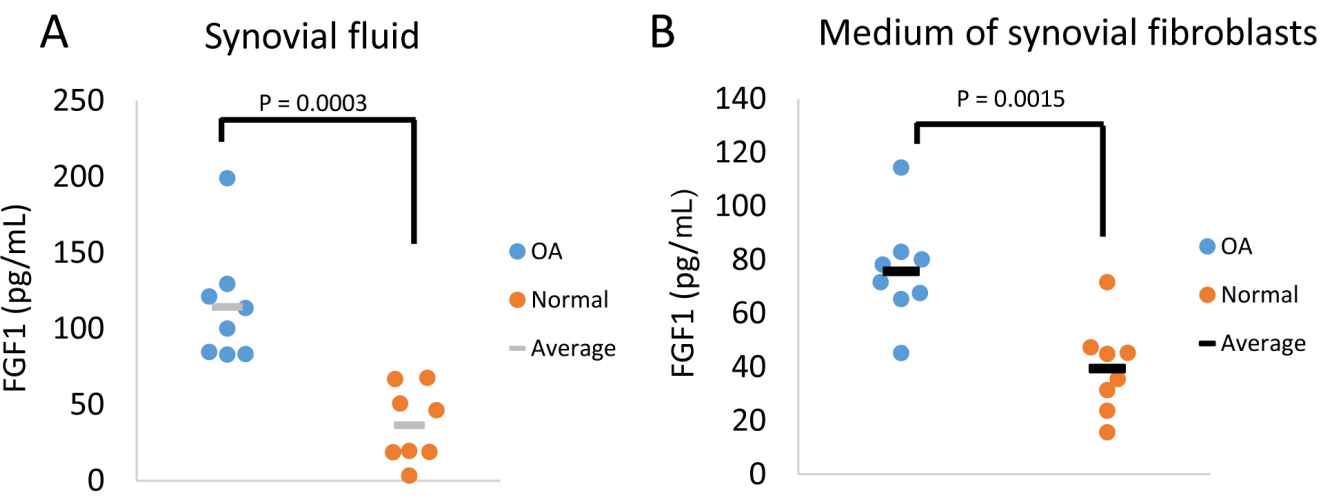

Figure 1. Concentrations of FGF-1 in the synovial fluid (A) and culture medium of synovial fibroblasts (B) from patients with OA or normal donors were measured by ELISA. Concentration is displayed as values from individual donors $(\bullet)$ as well as the average (-). OA, osteoarthritis. 
We then examined the expression of FGF-1 on the synovial membranes of patients with OA and normal donors by immunofluorescence staining. As shown in Figure 2 (left panels), segregated cells expressing FGF-1 were detected on the surfaces of synovial membranes from normal donors. Notably, FGF-1 expressing cells in OA synovial membranes were closer to each other and distributed deeper into the tissue compared to those in the normal sample. In general, the OA samples exhibited more FGF-1 staining than did normal samples. To further test if the expression of FGF-1 was subsequently lost during in vitro expansion, immunofluorescence staining for FGF-1 was also performed on cultured synovial fibroblasts at passage 2. Similarly, a greater number of positively stained cells were found in synovial fibroblasts isolated from patients with $\mathrm{OA}$ than in those from normal donors.
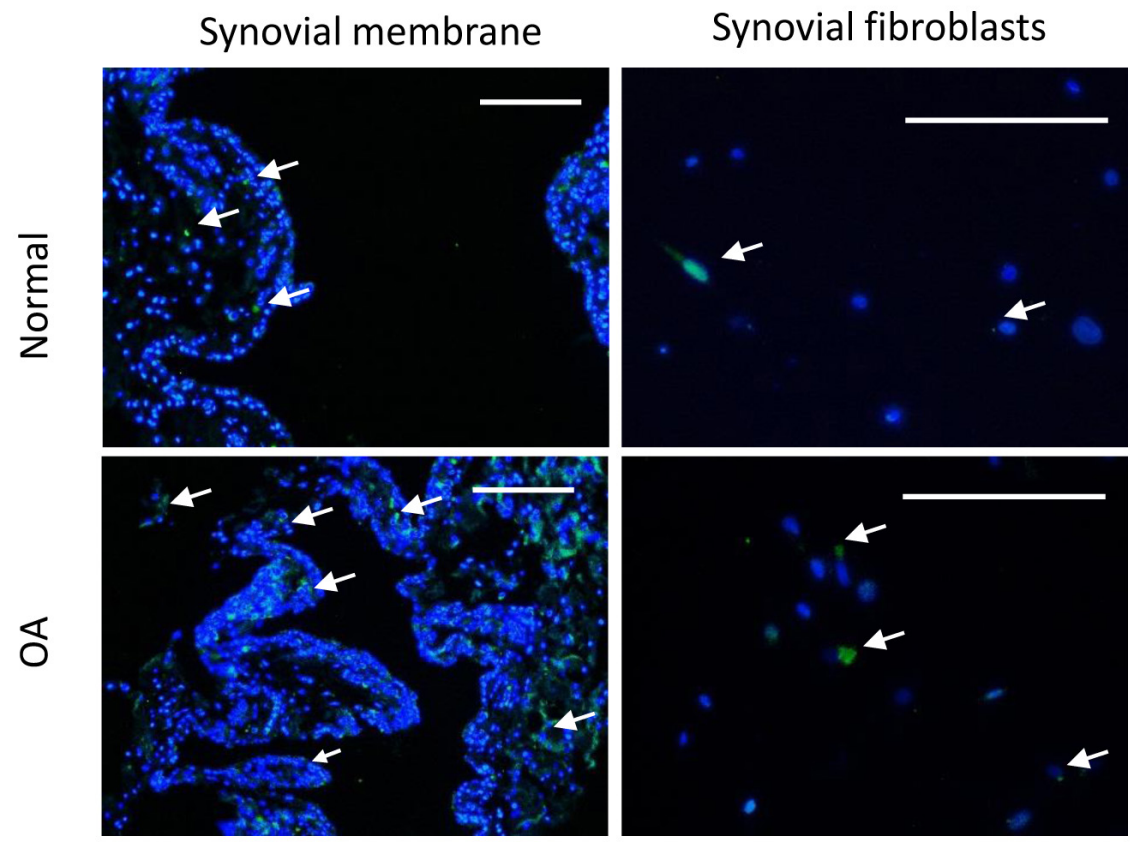

Figure 2. Immunofluorescence staining confirms the expression of FGF-1 in the synovial membranes of patients with OA and normal specimens. Cultured synovial fibroblasts also expressed FGF-1 at significant levels. Bar $=100$ $\mu \mathrm{m}$. Arrowheads indicate positive staining of FGF-1. OA, osteoarthritis.

The expression of $F G F-1$ at the mRNA level in normal and OA synovial membranes was then examined by qPCR. Expression of $F G F-1$ in the OA samples was found to be approximately 5 times greater than that in the normal samples (Figure 3 ). The difference between the normal and OA samples was statistically significant $(\mathrm{P}=0.0365)$. To further confirm the upregulation of FGF-1 signaling pathways in cartilage tissues, the expression of FGFR2 was also measured by qPCR. As shown in Figure 4, OA cartilage tissue expressed approximately two and a half times more $F G F R 2$ when compared to normal cartilage $(\mathrm{P}=0.0175)$. Nonspecific amplification was not detected in qPCRs for either FGF-1 or FGFR2, as is indicated by the single band on visualized on the agarose gel (Figure 5). 


\section{FGF1 in Synovium}

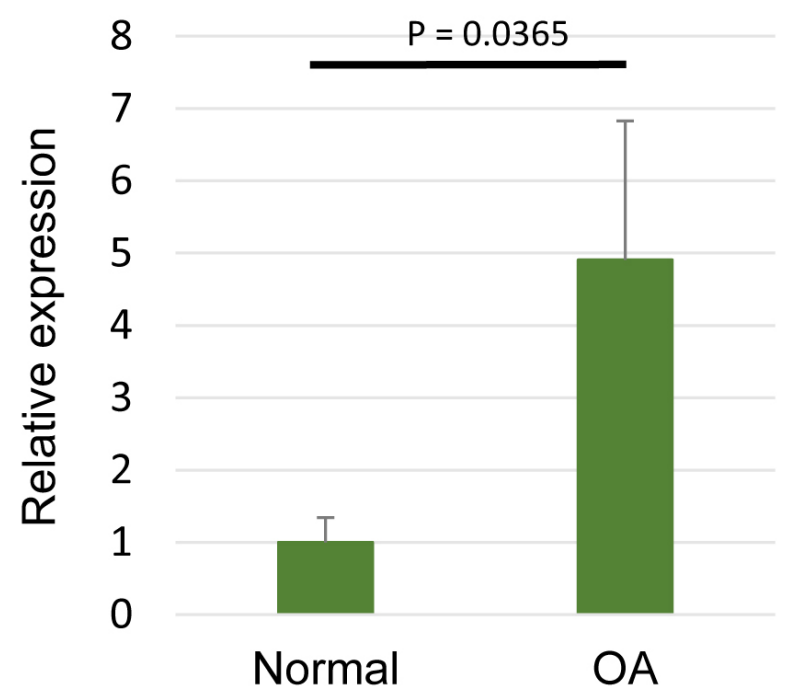

Figure 3. Real-time PCR indicated that the expression of $F G F-1$ in synovial membranes is upregulated in samples from patients with OA compared to those from normal controls. PCR, polymerase chain reaction; OA, osteoarthritis. Error bars represent standard deviation.

\section{FGFR2 in Cartilage}

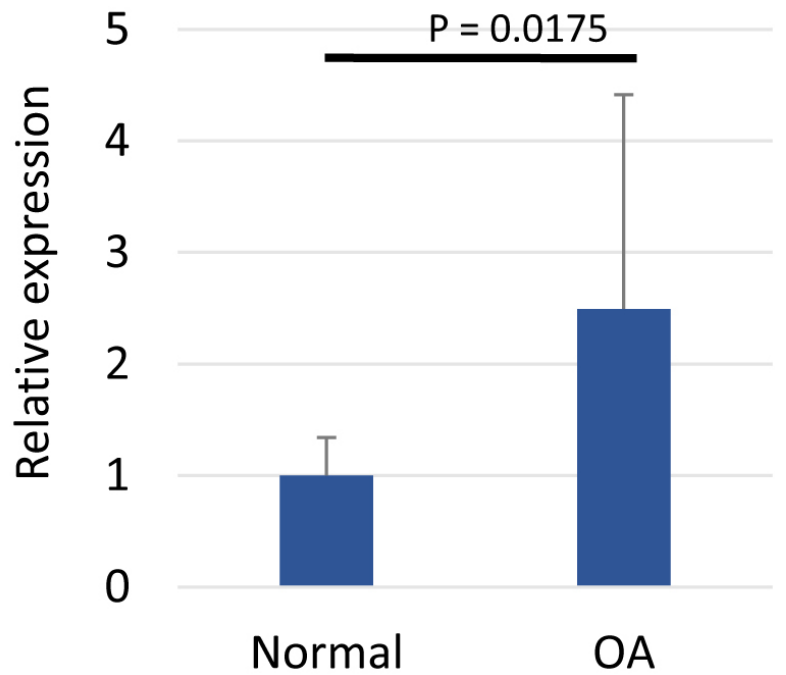

Figure 4. FGFR2 expression levels in samples of cartilage tissue from patients with OA and normal controls were examined by quantitative PCR. OA, osteoarthritis; PCR, polymerase chain reaction. Error bars represent standard deviation. 


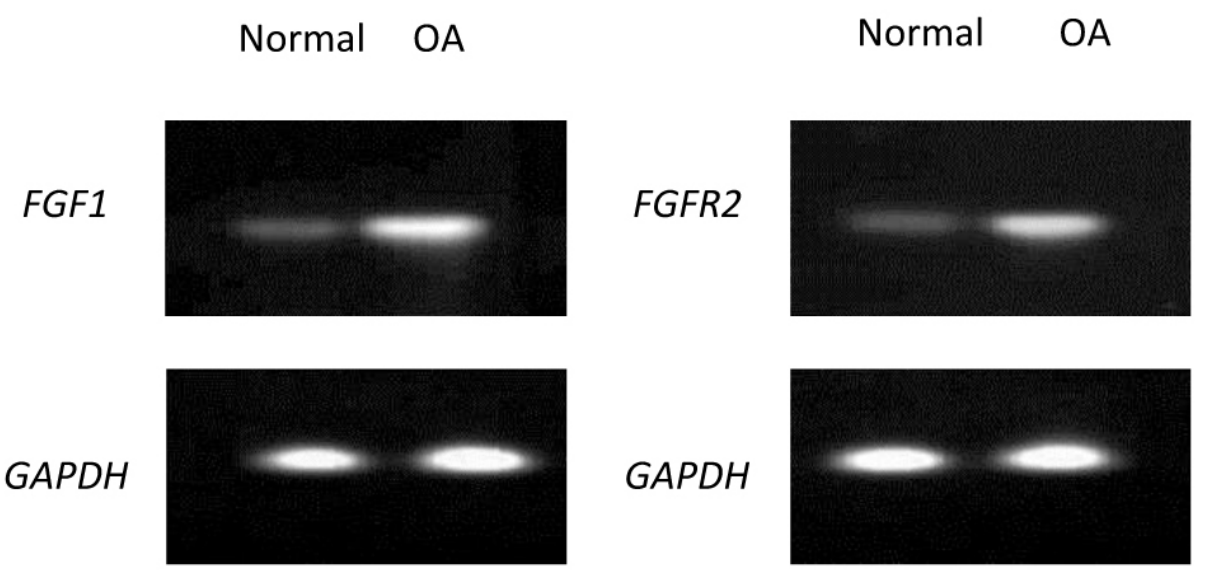

Figure 5. qPCR products were run on a $2 \%$ agarose gel to show the specificity of amplification. GAPDH is shown as the internal control. qPCR, quantitative real-time polymerase chain reaction; OA, osteoarthritis.

\section{DISCUSSION}

In this study, the concentrations of FGF-1 were measured in the synovial fluid and in the culture medium of synovial fibroblasts. Secretion and expression of FGF-1 were shown to be increased in the synovial membranes of patients with OA when compared to those of normal donors. The observation of higher expression of FGF receptors in OA cartilage than in normal cartilage confirmed that the overall FGF-1 signaling was upregulated under OA conditions.

FGF-1 was initially recognized as a mitogen for endothelial cells (Burgess and Maciag, 1989). It was then reported to stimulate cell proliferation for a variety of cell types including vascular smooth muscle, chondrocytes, osteoblasts, fibroblasts, and glial cells (Friesel and Maciag, 1999). FGF-1 was believed to be an angiogenic factor in vertebrate development and tissue repair, as well as being an inflammatory response factor in pathological conditions such as rheumatic arthritis (Thomas et al., 2000). This study was aimed at understanding the contribution of FGF to synovial pathology by examining its expression and secretion in synovial fluid and cultured synovial fibroblasts. We hypothesized that FGF production in synovial fibroblasts might be influenced by the OA environment. Our data supported this hypothesis by demonstrating higher expression and secretion of FGF-1 in OA samples.

It is believed that FGF expression might be specifically induced in inflammatory states such as rheumatic arthritis, as the level of FGF expression is often undetectable in noninflammatory tissues (Malemud, 2007). Here we showed that FGF-1 signaling is upregulated in OA as well as in rheumatic arthritis. This provides another piece of evidence to support that inflammation is closely related to the progression of OA. Several signaling pathways had been suggested to control the release of FGF-1 from cells into the extracellular environment. These include not only inflammation as previously mentioned, but also specific binding proteins (Czubayko et al., 1997), synaptotagmin-1 (Mouta Carreira et al., 1998), and serum starvation (Shin et al., 1996). Understanding the signals regulating FGF-1 secretion might be important for studying the mechanism underlying how OA is initiated after cartilage injury.

In conclusion, our data demonstrated that FGF-1 signaling is upregulated in OA pathological conditions. Our study represents the first report on FGF-1 expression and secretion 
in patients with OA. By comparing the secretion of FGF-1 using synovial fibroblasts, we provided evidence for an important role of FGF-1 signaling in the progression of OA. Further studies are in progress to study the role of FGF-1 in the angiogenesis induced by OA synovial fluid. Understanding these events might provide new targets for attenuating angiogenesis and the hypertrophic differentiation of chondrocytes in OA.

\section{Conflicts of interest}

The authors declare no conflict of interest.

\section{ACKNOWLEDGMENTS}

Research financially supported by the West China Hospital of Sichuan University through the Young Talent Research Program. The authors are grateful for the technical help from the Core Research Facility of the West China Hospital.

\section{REFERENCES}

Berenbaum F (2013). Osteoarthritis as an inflammatory disease (osteoarthritis is not osteoarthrosis!). Osteoarthr. Cartil. 21: $16-21$.

Bolander ME (1992). Regulation of fracture repair by growth factors. Proc. Soc. Exp. Biol. Med. 200: 165-170.

Böttcher RT and Niehrs C (2005). Fibroblast growth factor signaling during early vertebrate development. Endocr. Rev. 26: 63-77.

Burgess WH and Maciag T (1989). The heparin-binding (fibroblast) growth factor family of proteins. Annu. Rev. Biochem. 58: 575-606.

Chen GJ and Forough R (2006). Fibroblast growth factors, fibroblast growth factor receptors, diseases, and drugs. Recent. Pat. Cardiovasc. Drug Discov. 1: 211-224.

Czubayko F, Liaudet-Coopman ED, Aigner A, Tuveson AT, et al. (1997). A secreted FGF-binding protein can serve as the angiogenic switch in human cancer. Nat. Med. 3: 1137-1140.

Daheshia M and Yao JQ (2008). The interleukin 1beta pathway in the pathogenesis of osteoarthritis. J. Rheumatol. 35: 2306-2312.

Friesel R and Maciag T (1999). Fibroblast growth factor prototype release and fibroblast growth factor receptor signaling. Thromb. Haemost. 82: 748-754.

Itoh N and Ornitz DM (2004). Evolution of the Fgf and Fgfr gene families. Trends Genet. 20: 563-569.

Jackson A, Friedman S, Zhan X, Engleka KA, et al. (1992). Heat shock induces the release of fibroblast growth factor 1 from NIH 3T3 cells. Proc. Natl. Acad. Sci. U. S. A. 89: 10691-10695.

Livak KJ and Schmittgen TD (2001). Analysis of relative gene expression data using real-time quantitative PCR and the 2[-Delta Delta C(T)] Method. Methods 25: 402-408.

Malemud CJ (2007). Growth hormone, VEGF and FGF: involvement in rheumatoid arthritis. Clin. Chim. Acta 375: 10-19.

Mouta Carreira CM, LaVallee TM, Tarantini F, Jackson A, et al. (1998). S100A13 is involved in the regulation of fibroblast growth factor-1 and p40 synaptotagmin-1 release in vitro. J. Biol. Chem. 273: 22224-22231.

Shin JT, Opalenik SR, Wehby JN, Mahesh VK, et al. (1996). Serum-starvation induces the extracellular appearance of FGF-1. Biochim. Biophys. Acta 1312: 27-38.

Thomas JW, Thieu TH, Byrd VM and Miller GG (2000). Acidic fibroblast growth factor in synovial cells. Arthritis Rheum. 43: 2152-2159.

Wu L, Leijten J, van Blitterswijk CA and Karperien M (2013). Fibroblast growth factor-1 is a mesenchymal stromal cellsecreted factor stimulating proliferation of osteoarthritic chondrocytes in co-culture. Stem Cells Dev. 22: 2356-2367.

Zhen G and Cao X (2014). Targeting TGFbeta signaling in subchondral bone and articular cartilage homeostasis. Trends Pharmacol. Sci. 35: 227-236. 